Background Whereas the majority of STI-related consultations in the Netherlands take place in general practise (GP), national surveillance of STI predominantly uses data from STI centres, focussing at trends in high-risk groups. To also explore determinants of STI in the GP setting, an STI questionnaire was introduced in a nationwide GP-network.

Methods Since 2008, GPs of the Dutch Sentinel GP network (45 practises; 125,000 patients) are asked to complete a questionnaire for each STI-related episode, comparable to data collection in STI centres, and report laboratory results. Data included patient demographics, sexual behaviour and sex-life history.

Results Annually, for $0.4 \%$ of GP patients an STI consultation was recorded, mainly among young heterosexuals of Dutch origin, a profile comparable to STI centre visitors, though specific high-risk groups like MSM and CSW were reported less by GPs. GPs requested one or more laboratory tests in $83 \%$ of consultations; an STI was diagnosed in $34 \%$, most frequently chlamydia (21\%), condylomata $(9 \%)$ and herpes (6\%). Higher risk profiles were, depending on the STI: < 25 years old (chlamydia), MSM (gonorrhoea/syphilis), ethnic minorities (gonorrhoea), $>25$ years old (syphilis) or having symptoms (any STI). GP guidelines on multiple testing in high-risk groups (5 STI) were rarely fully adhered to, with many missed opportunities to test for HIV in patients with casual sexual contacts or originating from HIV-endemic countries.

Discussion STI consultation rates were lower than estimates based on electronic registers, probably due to underreporting. Patients who consulted a GP for STIs were comparable to persons attending STI-centres. Where STI-centres routinely test patients for chlamydia, syphilis, HIV and gonorrhoea, GPs test more selectively, resulting in higher case detection rates. This diverges from national GP guidelines and STI diagnoses may be missed. Opportunities for a more proactive role of GPs in STI and HIV testing should be explored.

\section{P3.035 PREVALENCE OF GENITAL MYCOPLASMAS AND BACTERIAL VAGINOSIS IN PREGNANT WOMEN IN GAUTENG, SOUTH AFRICA}

doi:10.1136/sextrans-2013-051184.0495

${ }^{1} \mathrm{M}$ J Redelinghuys, ${ }^{1} \mathrm{M}$ M Ehlers, ${ }^{1,2} \mathrm{~A}$ W Dreyer, ${ }^{3} \mathrm{H}$ Lombaard, ${ }^{1,2} \mathbf{M}$ M Kock 'Department of Medical Microbiology, University of Pretoria, Pretoria, South Africa; ${ }^{2}$ Tshwane Academic Division, National Health Laboratory Service, Pretoria, South Africa, ${ }^{3}$ Department of Obstetrics and Gynaecology, University of Pretoria, Pretoria, South Africa

Background Bacterial vaginosis and genital mycoplasmas are reproductive tract infections that are associated with several infections and adverse pregnancy outcomes, such as pelvic inflammatory disease, preterm birth and spontaneous abortions in affected women. Bacterial vaginosis (BV), a polymicrobial condition, is reported to be prevalent in $15 \%$ to $20 \%$ of pregnant women while mycoplasmas colonise up to about $70 \%$ of sexually active women and antenatal patients.

Methods Self-collected vaginal swabs were obtained from 221 pregnant women. Bacteria vaginosis was diagnosed using the Nugent scoring system and a multiplex PCR assay was performed to detect genital mycoplasmas. Mycoplasma hominis, M. genitalium, Ureaplasma urealyticum and U. parvum were targeted, respectively, for the $16 \mathrm{~S}$ rRNA gene, $140-\mathrm{kDa}$ adhesion protein and the multiplebanded antigen genes.

Results The prevalence of bacterial vaginosis was $17.6 \%(39 / 221)$ with a total of $23.1 \%$ (9/39) of all BV-positive cases (score 7 to 10) being HIV positive. An intermediate grading according to the Nugent system (score 4 to 6) was given to $14.9 \%$ (33/221) of samples. Ureaplasma parvum was isolated from the majority of samples with a prevalence of $72.4 \%(160 / 221)$ followed by $M$. hominis, which was present in $50.7 \%(112 / 221)$ of all samples. The prevalence of $M$. genitalium and
U. urealyticum was $14.5 \%(32 / 221)$ and $2.3 \%$ (5/221), respectively. Mycoplasma hominis was detected in $75 \%$ (27/36) of all HIV positive cases with U. parvum also present in 75\% (27/36) of these cases.

Conclusions The overall prevalence of genital mycoplasmas in antenatal patients was relatively high when compared to previous studies, while BV prevalence correlated with other reports. Ureaplasma parvum and $M$. hominis were notably present in HIV positive patients. The diagnosis of genital mycoplasmas and BV in pregnant women in HIV-prevalent South Africa is vital to allow early intervention and minimise complications, such as undesirable pregnancy outcomes.

\section{P3.036 GENITAL WARTS: DATA FROM THE ITALIAN GENERAL PRACTITIONERS}

doi:10.1136/sextrans-2013-051184.0496

'A Pasqua, ${ }^{2}$ S Costa, ${ }^{3} \mathrm{M}$ Salfa, ${ }^{4} \mathrm{~F}$ Lapi, ${ }^{3 B}$ Suligoi. ${ }^{1}$ Health Search - Italian College of General Practitioners, Florence, Italy; ${ }^{2}$ University of Bologna, Bologna, Italy; ${ }^{3}$ stituto Superiore di Sanità, Rome, Italy; ${ }^{4}$ talian College of General Practitioners, Florence, Italy

Background Worldwide, several million cases of genital warts occur each year. The objective of this study was to estimate the incidence of genital warts (GW) in Italy, in a sample of the general population aged $15-64$ years.

Methods A retrospective study was conducted in 2009 with the collaboration of 650 general practitioners (GPs), geographically representative of all Italian GPs.

Cases of GW were identified as follows: (1) individuals diagnosed with the ICD-9 code 078.11 (genital warts); or (2) individuals diagnosed with the ICD-9 code 078.10 (warts) who were prescribed podofillin/imiquimod treatment or were referred to a specialist (gynaecologist/dermatologist/urologist). Incidence was calculated using the number of persons included in the GPs lists as denominator. Individual data on age, gender, and area of residence were available.

Results The lists of the 650 GPs included 775,644 persons; their median age was 48 years, $52.7 \%$ were women. The incidence of GW was $0.44 \%$ (95\% CI $0.39-0.49)$; specifically, $0.41 \%$ among men $(95 \%$ CI $0.36-0.47)$ and $0.51 \%$ among women (95\% CI $0.43-0.57)$. The highest incidence was observed among 25-34 year-old individuals (0.64\%; $95 \%$ CI $0.52-0.78)$, for both genders. When stratifying by geographical area, the incidence was $0.40 \%$ in Northern Italy, $0.32 \%$ in Central Italy and $0.45 \%$ in Southern Italy.

Conclusions This is the first study to provide an estimate of the incidence of GW in the Italian population attending GPs. The incidence observed is similar to that reported by GPs in the UK (Desai S, 2011; Cassel JA, 2006). These results show that GPs can have a key role in preventing the spread of GW by providing early diagnosis and appropriate referral, especially among young persons, women and in persons living in Southern Italy.

\section{P3.037 HIGH PREVALENCE OF GENITAL WARTS AMONG YOUNG WOMEN IN ITALY}

doi:10.1136/sextrans-2013-051184.0497

${ }^{1} \mathrm{~L}$ Mariani, ${ }^{2} \mathrm{M}$ Salfa, ${ }^{3} \mathrm{~L}$ Timelli, ${ }^{4} \mathrm{G}$ Vittori, ${ }^{5} \mathrm{G}$ Fattorini, ${ }^{2} \mathbf{B}$ Suligoi. ${ }^{1}$ Regina Elena National Cancer Institute, HPV-Unit, Rome, Italy; 'Istituto Superiore di Sanità, Rome, Italy; ${ }^{3}$ nforma srl, Rome, Italy; ${ }^{4}$ Ospedale San Carlo di Nancy, Rome, Italy; ${ }^{5}$ Azienda Sanitaria di Bologna, Bologna, Italy

Background Worldwide, several million cases of genital warts occur each year. The objective of this study was to estimate the prevalence and the incidence of external genital warts (eGW) in a sample of women attending gynaecological ambulatories and to estimate the number of women with eGW among women aged 15-64 years in Italy 